\title{
A New Approach of Designing a Client/Server Model with Smart Screen
}

\author{
Dheyaa Jasim Kadhim \\ University of Baghdad \\ Electrical Engineering Department
}

\author{
Muntader M. Jasim \\ University of Baghdad \\ Computer Engineering Department
}

\begin{abstract}
Advances in technology make it possible for network users to experience personalized services anytime and anywhere. But this can only take place if our network systems have well designed networks that support an increasing number of devices can be controlled and monitored. So what are the ideal ways to replace or update existing networks, control, develop and support new network infrastructures, and provide reliable and secure access. Build a smart network (client server model) compound from a server connected with a smart terminal client consists of a virtual display with mouse and keyboard only, this new design of network different from the conventional network design (client server model) in many aspects. The new design enhances the performance of the classical (conventional) client server model in sides of security, reducing power consumption, cost, used resources, and software and hardware issues.
\end{abstract}

\section{Keywords}

Smart Network, Client-Server Model and Conventional Network Design.

\section{INTRODUCTION}

Computer networks can be used for numerous services; both for companies and for individual. For companies, networks of personal computers using shared servers often provide flexibility and good price/performance ratio. For individuals, networks offer access to a variety of information and entertainment resources [1].

For connected terminals of network and the networks together can use twisted pair, coax, and fiber optics but for people who need be on line all time, for these mobile users, need to get their hits of data for their laptop, notebook, shirt- pocket, palmtop, or wristwatch computers without being tethered to the terrestrial communication infrastructure, for these users, wireless communication is the intended target [2].

Computer networks can be classified according to provide services as Client-Server model and Peer-to-Peer model. Traditional the most used model in networks are the ClientServer model which is considered as a popular model for computer networks since the clients and servers devices are specifically designed for special purposes as networks requirements. The Client-Server model can be applied on the organizations, universities, companies and other establishments using the Internet as well as any internetwork technologies. Examples services applied client-server model on the Internet include Web browsers and Web servers, FTP clients and servers, DHCP, POP3 and DNS [1][3][4].

Figure 1 shows the Client-Server model which is spreading dramatically in many years ago since the personal computers (PCs) and the laptops became the common alternative to older mainframe computers. Client devices are typically personal computers that deal with some application needed specified network software that achieves the required application through requesting and receiving information over the network. Mobile devices (smart phones or laptops) as well as desktop computers can both function as clients [5].

A server is a special device which used to store files and huge database including more complex applications like web sites, streaming video, online game, communication services...etc. Server devices often feature higher-powered central processors and larger disk drives than clients [6].

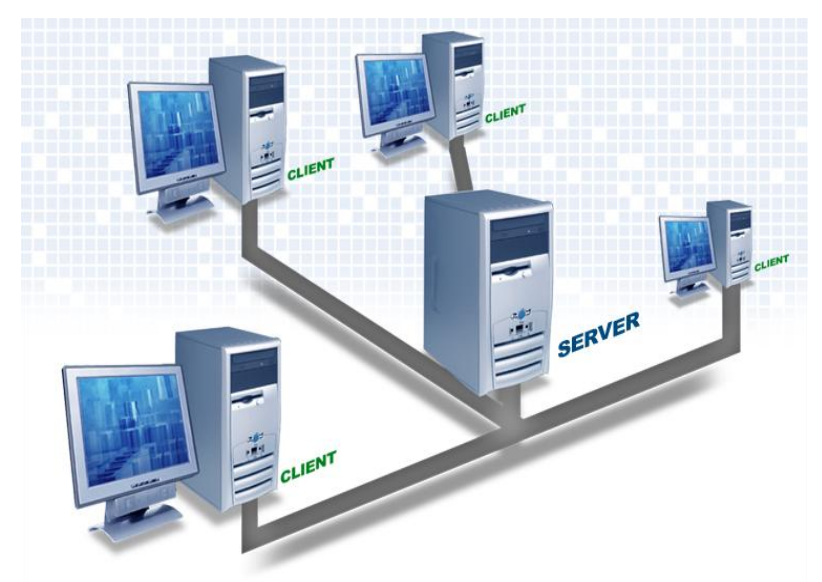

Fig. 1: Client-Server Model

In this work, a new approach of client-server model based smart behavior is proposed and applied to compound a smart network from virtual display with mouse and keyboard only instead of standalone PC (with monitor, case unit that contains processor, RAM, mother board...etc.).

This paper organized as follows: section 2 will describe the most important applications for client-server model; section 3 gives the whole description of our proposed smart network model; section 4 shows the results of the proposed work in this paper and discusses these results deeply. Finally section 5 gives the aggregated conclusions from our work abd gives also the suggested future works.

\section{CLIENT-SERVER APPLICATIONS}

The client-server model deals with different applications according to the clients requests. Network users send make requests to a specified server by sending messages, and these servers respond to their users or clients by expressing on each request and returning results. Each specified server generally supports many clients, and multiple servers can be grouped together in a pool to deal with the dramatic processing load as the number of clients increases [7]. 
A client device and a server device are usually two separate devices, each characterized for their designed purpose. For example, a Web client can browse as much as screen display being large, whereas a Web server does not need screen display at whole and can be found anywhere in the world. However, in some cases a given device can function both as a client and a server for the same application. Likewise, a device that is a server for one application can simultaneously act as a client to other servers, for different applications [8][9].

Traditionally, the most popular applications on the Internet consider the client-server model including web site, file transfer, video streaming, online game and more other services. The clients of all these services use a specified interface to access them (either graphic- or text-based) and then clients' applications will allow the users to connect to servers. In the case of email and FTP applications, clients enter a computer name (or sometimes an IP address) into the interface to install a communication link to the server [10].

Nowadays, many popular networks use client-server models without even realizing it. Access routers in our home for example, contain DHCP servers that provide IP addresses to our PCs and smart phones (DHCP clients). Other types of network servers found in companies include print servers and backup servers [11].

\section{PROPOSED MODEL}

Comparing between conventional network (client-server model as be shown in Figure 1 with the smart network design model that can be shown in Figure 2 below, our proposed smart network design can provide performance enhancement, Quality of services, reduce power consumptions, support software and hardware issues and any other advantages can get it from the proposed smart mode.

The conventional model consists of one server by using Dell power edge T1100 server, 8-port hub switch D-link, 4standalone PC's (each PC with 2GB RAM and processor core due $2.3 \mathrm{GHZ}$ with built in Ethernet connection motherboard) and traditional monitor with mouse and keyboard, the network connected with UTP cable category 5.

While the smart network design model can be shown in Figure 2, consists of the same server and hub switch used in conventional model above 4-smart virtual display (a display has a built-in Ethernet connection, USB's ports) with mouse and keyboard only connected directly to the smart virtual display instead of the case and the traditional monitor which we used in the conventional model described above.

These two models are practically designed and implemented in lab and will be put under consideration to test their performance according to some important performance metrics such as data transfer rate, security issue, aggregated implementation cost, power consumption, resources used and finally how much required software to work with.

To test these two models, important applications should be used and put these two models under consideration and prove their abilities and responsibilities to show which one can give better performance evolution and can be heavy duty through hard work and heavy load specially they represent central systems or on the other hands client-server systems.

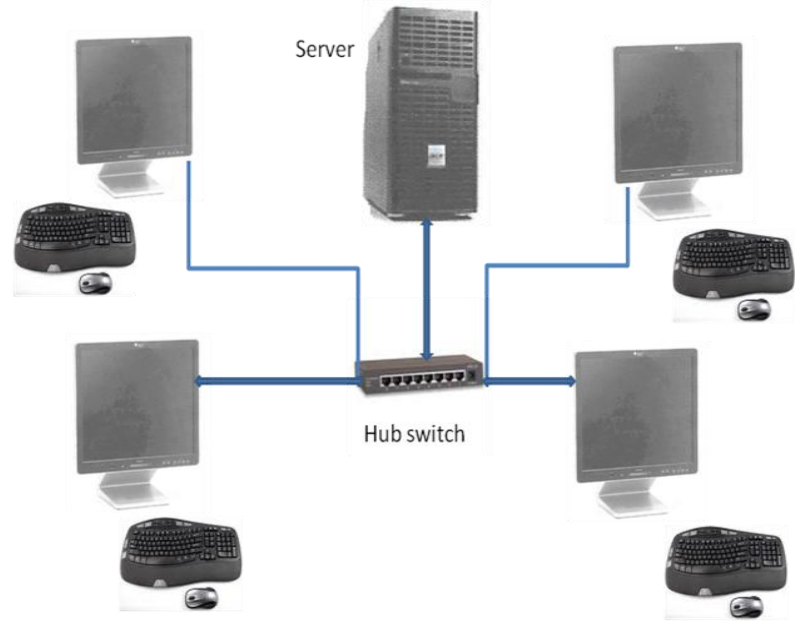

Virtual Display with keyboard and mouse

Fig. 2: Proposed Smart Client-Server Model

\section{RESULTS AND DISCUSSION}

From our work we found that the smart model performance is higher than what we obtained in the conventional model from the prospect of data transfer, used resources, power consumption, security issues, cost, and software issues.

The data transfer in smart model dependents on the server performance, wire network properties and the Ethernet connection in smart virtual display comparing with presence of case unit that used in conventional model.

The smart design model showed in Figure 2, consists of the same server and hub switch used in conventional model above 4-smart virtual display (a display has a built-in Ethernet connection, USB's ports) with mouse and keyboard only connected directly to the smart virtual display instead of the case and the traditional monitor which we used in the conventional model described above

The used resources with smart model is less than the resources used in conventional model; in the smart model we just use a smart virtual display with mouse and keyboard only for each client instead of standalone PC (with monitor, case unit that contains processor, RAM, mother board...etc.).

It's clear that the power consumption in the smart model is less than in the conventional model due to fewer resources used and the efficiency of using UPS units.

The smart model has high security due to the data is located in the server only (centralized system) and the server gives the clients permissions to access to a certain date (read / write permissions).

The cost of building the smart model is less than the conventional model due to hardware and software cost.

The required software used in the smart model installed only on the server and the clients use it virtually and separately.

Table 1 and Figure 3 show a comparison between the conventional model for one server with four clients and the proposed smart model. While Table 2 gives the general performance differences between these two models according to some performance matrices such as (data transfer, security, power consumption, cost, used resources and required software). 
Many applications have to use through this proposed smart design model such as laboratories and campuses for scientific research, factories for control and management purposes, offices to support data and resources sharing, hospitals and medical care centers for tracking and updating the patient's records and finally it can be used for E-Learning and Egovernment applications.

Table 1. Comparison between Conventional Model and Proposed Smart Design Model

\begin{tabular}{|l|c|c|}
\hline Component & $\begin{array}{c}\text { Conventional } \\
\text { Model }\end{array}$ & $\begin{array}{c}\text { Proposed Smart } \\
\text { Model }\end{array}$ \\
\hline Hard Disks & 5 & 1 \\
\hline Software & 5 & 1 \\
\hline Power Sources & 10 & 5 \\
\hline Motherboards & 5 & 1 \\
\hline RAM's & 5 & 1 \\
\hline Total No. & 30 & 9 \\
\hline
\end{tabular}

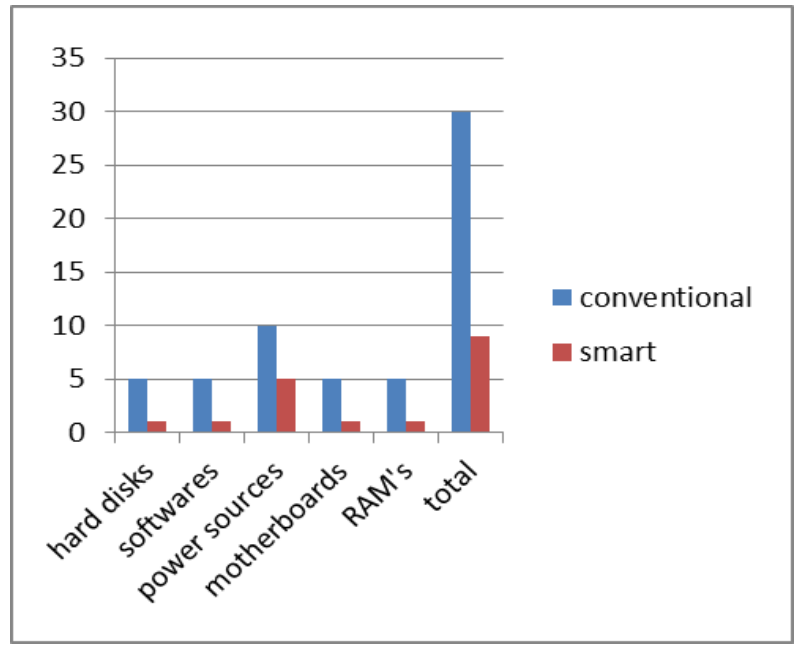

Fig. 3: Comparison Chart between Conventional Model and Proposed Smart Design Model

Table 2. General Performance Differences between Conventional Model and Proposed Smart Design Model

\begin{tabular}{|l|c|c|}
\hline \multicolumn{1}{|c|}{ Metrics } & Smart Model & $\begin{array}{c}\text { Conventional } \\
\text { Model }\end{array}$ \\
\hline Data transfer & High & Low \\
\hline Security & High & Low \\
\hline Cost & Low & High \\
\hline $\begin{array}{l}\text { Power } \\
\text { Consumption }\end{array}$ & Low & High \\
\hline Used Resources & Less & More \\
\hline $\begin{array}{l}\text { Required } \\
\text { Software }\end{array}$ & Less & More \\
\hline
\end{tabular}

\section{CONCLUSIONS \& FUTURE WORKS}

Comparing between the two models the conventional and the smart design leads us to see the advantages of smart model in different issues (power consumption, hardware, used resources, security, software and cost) but not in all applications we can prefer this design to conventional one, in some applications the client need a standalone pc to use it separately, if he need a CD room or a special application to be installed on its PC only; so that, the choice of which model we need, depend upon the kind of application that the model used for.

A number of future research topics were identified and the following is a suggested list of possible research directions:

1. Using a wireless or an optical fiber connection in the smart model for connecting the clients to the server instead of the wireline connection used in the conventional model.

2. Using a new generation of the smart screens which provides advanced features like touch screen, card reader and multi USP port.

3. Connecting the servers together via WAN to support data exchanging and file sharing among connected servers.

4. Connecting the server to the internet to provide variety services to the clients.

\section{REFERENCES}

[1] Andrew S. Tanenbaum and David J. Wetherall, 2010. Computer Networks, $5^{\text {th }}$ edition, Prentice Hell.

[2] William Stallings. 2013. Data and Computer Communications. $10^{\text {th }}$ edition, Prentice Hell.

[3] Alex Berson.1996. Client/Server. 2nd edition. McGrawHill.

[4] Patricia A. Goglia. 1993. Testing Client/Server Applications. Wiley-QED Publication.

[5] Chad Yoshikawa and etal. 1997. Using Smart Clients to Build Scalable Services. Computer Science Division, University of California, Berkeley.

[6] Chutimet Srinilta and Alok Choudhary. 1998 Performance Enhancement Using Intra-server Caching in a Continuous Media Server. Proceedings of 8th International Workshop on Research Issues in Data Engineering.

[7] Mark Boulter. 2004. Smart Client Architecture and Design Guide. Microsoft ${ }^{\circledR}$ patterns \& practices Library.

[8] Gary M. Weiss and Jeffrey W. Lockhart. 2012. A Comparison of Alternative Client/Server Architectures for Ubiquitous Mobile Sensor-Based Applications, Proceedings of the 2012 ACM Conference on Ubiquitous Computing.

[9] Johan Bollen and Michael L. Nelson,2002. 31st International Conference on Parallel Processing Workshops (ICPP 2002 Workshops), Vancouver, BC, Canada.

[10] John Mcintyre and Dan Squillace. 1994. Implementing Client/Server Applications in a Heterogeneous Platform Environment. WUSS.

[11] Jahangir Karimi. 1999. A software design technique for client-server applications. Concurrency: Practice and Experience Journal, Vol. 11(1), 21-42. 